This item was submitted to Loughborough's Research Repository by the author.

Items in Figshare are protected by copyright, with all rights reserved, unless otherwise indicated.

\title{
Effect of exercise and heat-induced hypohydration on brain volume
}

PLEASE CITE THE PUBLISHED VERSION

http://dx.doi.org/10.1249/MSS.0b013e3181e39788

\section{PUBLISHER}

Lippincott, Williams \& Wilkins (๔ American College of Sports Medicine)

\section{VERSION}

AM (Accepted Manuscript)

\section{LICENCE}

CC BY-NC-ND 4.0

\section{REPOSITORY RECORD}

Watson, Phillip, Kay Head, Alain Pitiot, Peter Morris, and Ronald J. Maughan. 2019. "Effect of Exercise and Heat-induced Hypohydration on Brain Volume". figshare. https://hdl.handle.net/2134/11373. 
This item was submitted to Loughborough's Institutional Repository (https://dspace.lboro.ac.uk/) by the author and is made available under the following Creative Commons Licence conditions.

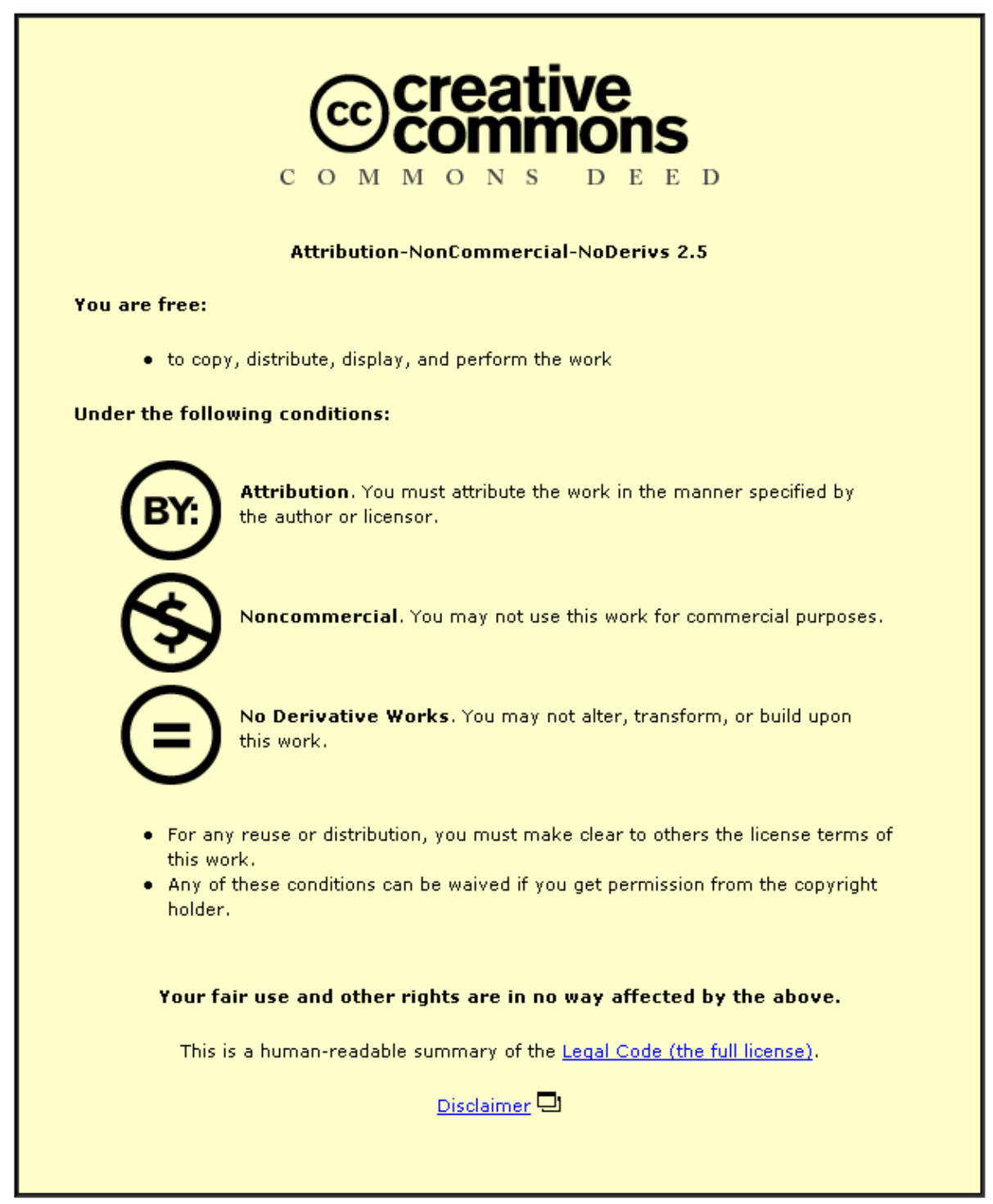

For the full text of this licence, please go to: http://creativecommons.org/licenses/by-nc-nd/2.5/ 
Effect of exercise and heat-induced hypohydration on brain volume

Phillip Watson ${ }^{1}$, Kay Head ${ }^{2}$, Alain Pitiot ${ }^{3}$, Peter Morris ${ }^{2}$ and Ronald J. Maughan ${ }^{1}$

${ }^{1}$ School of Sport, Exercise and Health Sciences, Loughborough University,

Leicestershire, LE11 3TU, UK.

${ }^{2}$ Sir Peter Mansfield Magnetic Resonance Centre, School of Physics and Astronomy, University of Nottingham, University Park, Nottingham, NG7 2RD, UK.

${ }^{3}$ Brain and Body Centre, School of Psychology, University of Nottingham, University Park, Nottingham, NG7 2RD, UK.

Correspondence: $\quad$ Professor Ronald J Maughan,

School of Sport and Exercise Sciences,

Loughborough University,

Leicestershire,

LE11 3TU,

United Kingdom.

Telephone: $\quad$ +44 (0)1509 226329

Fax: $\quad$ +44 (0)1509226301

Email: $\quad$ r.j.maughan@lboro.ac.uk

Running title: Hypohydration and brain volume

Disclosure of funding: This study was funded by the Gatorade Sport Science Institute (GSSI), Barrington, Illinois, USA. 


\section{Abstract}

Purpose: The aim of the present study was to quantify changes in brain volume following exercise/heat-induced hypohydration in man. Methods: Eight active men completed intermittent exercise in a warm environment, until $2.9 \pm 0.1 \%$ of body mass was lost. Subjects remained hypohydrated for two hours following the end of exercise. Brain volume was measured before, immediately following, and $1 \mathrm{~h}$ and $2 \mathrm{~h}$ after exercise using MRI (Philips 3T Achieva). Measures of subjective feelings and core body temperature were also monitored. Blood samples were drawn to determine serum electrolyte concentrations and osmolality and to allow calculation of changes in blood and plasma volumes. Results: Brain volume was not influenced by hypohydration $(0.2 \pm 0.4 \%$; ES 0.2; $\mathrm{P}=0.310)$. Reductions in ventricular $(4.0 \pm 1.8$ \%; ES 4.6; $\mathrm{P}<0.001)$ and CSF (3.1 $\pm 1.9 \%$; ES 3.3; $\mathrm{P}=0.003)$ volumes were observed following exercise. Compared with pre-exercise levels, serum osmolality was elevated throughout the $2 \mathrm{~h}$ post-exercise period $(+10 \pm 2 \mathrm{mosmol} / \mathrm{kg} ; \mathrm{P}<0.001)$. Core temperature increased from $37.1 \pm 0.3^{\circ} \mathrm{C}$ at rest to $39.3 \pm 0.5^{\circ} \mathrm{C}$ at the end of exercise $(\mathrm{P}=0.001)$. Conclusions: These data demonstrate that brain volume remains unchanged in response to moderate hypohydration and the presence of serum hyperosmolality, suggesting that mechanisms are in place to defend brain volume.

Keywords: dehydration; hyperosmolality; cerebrospinal fluid; MRI 


\section{Introduction}

Paragraph 1 A common practice used by participants in weight-category sports is to achieve an acute loss of body mass prior to competition. This might be to compete at a lower weight category (boxing, martial arts), or to comply with weight penalties employed in some sports (e.g. horse racing). Athletes in weight-category sports tend to display higher morning urine osmolality values than those observed in the general population, perhaps reflecting their attempts to maintain a low body mass through dehydration (17). A survey of Australian jockeys found that $81 \%$ restricted food and fluid intake in the $24 \mathrm{~h}$ prior to competition, with exercise/sauna-induced sweating and diuretic use also regularly employed to reduce body mass (12). These practices all result in a progressive loss of body water, causing an increase in plasma osmolality. This in turn produces a movement of water from interstitial and intracellular spaces to defend blood volume, thus helping to maintain blood flow to the exercising muscles and to the skin (13). While this response is well defined in the periphery, it is also clear that an increase in extracellular osmolality can exert an influence on total brain (3) and brain cell (1) volumes, potentially also influencing the permeability of the blood-brain barrier (BBB) (25).

Paragraph 2 At present there are limited data on the effect of dehydration on the central nervous system (CNS) in humans. Recent work has employed Magnetic Resonance Imaging (MRI) to determine the effects on brain volume of dehydration induced by exercise under conditions of restricted heat loss $(5,10)$ and during a 16 hour period of fluid restriction (7). Interestingly, while changes in brain volume were reported following fluid-restriction protocols, no change was observed following 
exercise-induced dehydration. The two previous reports investigating the response to exercise have reported marked changes in ventricular volume, with the magnitude of change loosely related to the degree of hypohydration induced $(5,10)$. The reasons for the apparent discrepancy between these passive and active forms of dehydration are unclear, particularly as the magnitude of total body water loss, expressed as a percentage reduction in body mass, was greater in the exercise studies (Passive $1.6 \pm$ $1.0 \%$; Active $2.3 \pm 0.2 \%$ and $2.2 \pm 0.5 \%$ ). It is possible that the gradual loss of fluid over a prolonged timescale with fluid restriction produces a different response from that which occurs with rapid loss of fluid caused by exercise in the heat, but this can only be speculated. Animal data suggest that any loss of fluid from the brain results in the rapid movement of osmolytes from the cerebrospinal fluid (CSF) and serum into the brain to defend brain volume and limit further fluid losses (8). Water loss and electrolyte uptake are likely to occur almost simultaneously, with the magnitude of brain water losses demonstrated to be only $35 \%$ of that anticipated in the $30 \mathrm{~min}$ following osmotic opening of the blood-brain barrier (3). It is possible that redistribution of fluids to restore brain volume might have confounded the results of some studies investigating the effect of exercise and thermally-induced fluid losses $(5,10,14)$.

Paragraph 3 In some sports there can be a long interval between the weigh-in and the start of competition (e.g. professional boxing). This movement of fluids might protect against potential changes in brain volume resulting from the dehydration practices employed by competitors in these sports. There are situations, however, where competition begins only a few minutes following a weigh-in (horse racing, amateur boxing/martial arts): this, coupled with any hypohydration accrued during the activity 
itself, might place these individuals at an increased risk of traumatic brain injury. A marked reduction in the size of the brain has the potential to increase trauma experienced during falls and collisions because of greater movement of the brain in the skull after impact. Apart from the risk of intracranial bleeding, collisions between the surface of the brain and the walls of the interior skull might cause the cortical tissue to be deformed, compressed or stretched and this has been implicated in the aetiology of concussion (18). Clearly the high risk of falls or blows to the head in some sports can place competitors at risk of traumatic brain injury. Whether practices routinely employed to achieve a desired competition weight can affect brain volume is therefore an interesting question. This is true from a purely mechanistic standpoint, but might also have implications for sports medicine, as the number of concussions and other head injuries sustained by competitors in weight-category sports appears to be greater than reported in other sports $(2,23)$.

Paragraph 4 The aim of the present study was to examine the effect of exerciseinduced hypohydration on brain, ventricular and CSF volumes. Previous studies examining changes in brain volume with dehydration have reported inconsistent results, particularly in the changes observed in ventricular volume. These investigations have employed varying levels of dehydration, which could explain some of the inconsistency in this response. In addition, there has yet to be any examination of the potential cell-volume regulation response in humans that has been reported in animals to defend brain volume in the presence of an osmotic challenge. To achieve this, every effort was made to complete the first measurements as soon as possible after the cessation of exercise. In addition, responses were observed over a 
two-hour period after exercise where no fluid was provided to examine a time course of change.

\section{Materials and Methods}

Paragraph 5 Subjects. Eight physically active men (Mean \pm SD age $26 \pm 4$ y; height $1.79 \pm 0.09 \mathrm{~m}$; body mass $79.7 \pm 11.2 \mathrm{~kg}, \mathrm{VO}_{2}$ peak $4.1 \pm 0.3 \mathrm{~L} / \mathrm{min}, \%$ body fat 12.2 $\pm 1.6 \%$ ) were recruited to take part in this investigation. All subjects were physically active and habituated to the sensation of strenuous exercise, but none was accustomed to exercise in a warm environment at the time of the study. Prior to volunteering, all subjects received written details outlining the nature and purpose of the study. Following any questions regarding the protocol, a written statement of consent was signed. The protocol was approved by the Ethical Advisory Committees of Loughborough University and the University of Nottingham.

Paragraph 6 Experimental Protocol. All subjects completed a preliminary test to measure peak oxygen uptake $\left(\mathrm{VO}_{2}\right.$ peak), a familiarisation trial and a single experimental trial. $\mathrm{VO}_{2}$ peak was determined using a discontinuous, incremental exercise test to volitional exhaustion on a cycle ergometer (Monark Ergomedic 824E). The familiarisation trial involved the completion of the same exercise protocol as the experimental trial. The familiarisation and experimental trials were separated by at least 7 days to limit the development of heat acclimation. Subjects were instructed to record dietary intake and physical activity during the day prior to the familiarisation trial, and to replicate this before the subsequent experimental trial. No strenuous 
exercise or alcohol consumption was permitted for 24 hours before each visit to the laboratory.

Paragraph 7 Dehydration Protocol. The trials took place in the morning following an overnight fast, other than the ingestion of $500 \mathrm{ml}$ of plain water $90 \mathrm{~min}$ before commencing exercise. On arrival at the laboratory, subjects first emptied their bladder, and a sample of urine was retained for measurement of osmolality (Gonotec Osmomat 030, YSI, Farnborough, UK). Subjects then sat in a comfortable environment $\left(22-24{ }^{\circ} \mathrm{C}\right)$ for 20 min before entering a climatic chamber maintained at a temperature of $35.1 \pm 0.4{ }^{\circ} \mathrm{C}$ and relative humidity of $57 \pm 3 \%$ where nude body mass was measured to the nearest 10 g (Adam CFW150 digital scale, Milton Keynes, UK). The subject then began a series of 10 min periods of cycle exercise at an intensity corresponding to $60 \%$ of $\mathrm{VO}_{2}$ peak. A 5 min rest period separated successive blocks of exercise, during which the subject towelled dry and nude body mass was recorded. Sweat losses were determined for each period of exercise through changes in body mass, and this pattern of activity and rest continued until the subject had lost approximately 3\% of their initial body mass. Subjects then returned to a comfortable environment, and were not permitted to ingest any fluid for two hours following the end of exercise.

Paragraph 8 Measures: Because of limitations associated with the monitoring of core temperature when exposed to MRI, this was measured during the familiarisation trial. A radio-telemetry pill (HQ Inc, Palmetto, Florida) was ingested 10 hours prior to this trial, before an overnight fast, to enable intestinal temperature to be determined. The subjective feelings of thirst, alertness and fatigue were assessed using a series of 
$100 \mathrm{~mm}$ visual analogue scales before exercise, immediately following exercise and during the 2 hour recovery period.

Paragraph 9 To determine the normal variation and/or test-retest error in measured brain and CSF volumes over the same timeframe as the experimental trials, two subjects, who did not participate in the subsequent trials, remained at rest in a comfortable environment over a three hour period. During the experimental trial measurements of brain, ventricular and CSF volume were undertaken prior to exercise, upon attainment of $3 \%$ body mass loss, and one and two hours after the end of exercise. Anatomical MPRAGE and Vista scans were performed at each time point using a 3 Tesla magnetic resonance scanner (Philips Achieva, AE Eindhoven, The Netherlands). Imaging parameters were as follows: MPRAGE: TE $2.3 \mathrm{~ms}$, TR $7.7 \mathrm{~ms}$, voxel size 0.8 x 0.8 x 0.8 mm, 256x256 matrix, 205x205x147mm field of view; Vista (this scan specifically highlights the CSF and was used in volumetric method 2 described below): TE 380 ms, TR 2500 ms, voxel size 0.8 x 0.8 x 0.8 mm, 312x312 matrix, 250x250x180 field of view.

Paragraph 10 The brain in all scans was first extracted using the BET skull stripping tool (19). Brain-extracted images were then inspected, to ensure any residual nonbrain tissue (neck tissue, nasal sinus, skull etc) had been removed. All input scans were transformed to the centre position of the set, to ensure a homogeneous amount of blurring related to interpolation, using robust linear registration (15). Percentage changes in brain, ventricular and CSF volumes were then estimated using two methods. Volumetric Method 1: changes in brain volume were determined from the MPRAGE scans using SIENA (version 2.6, FMRIB Software Library, Oxford 
University, UK), a fully automated analysis tool designed for the longitudinal assessment of brain atrophy $(20,21)$. SIENA segments the brain images to determine the brain/CSF edge points, with the perpendicular displacement of these edge points used to estimate percentage brain volume change between scans. Regional brain volume changes were determined using the voxelwise extension of SIENA: the edge displacement images generated for each subject were spatially dilated, transformed to a standard space, masked with a standard brain edge image (MNI152) and smoothed by a Gaussian kernel of $5 \mathrm{~mm}$. Changes in ventricular volume were determined by manually removing the non-ventricular CSF prior to tissue-type segmentation undertaken using FAST (FMRIB Software Library, Oxford University, UK) to determine the brain/non-brain boundaries (27).

Paragraph 11 Volumetric Method 2: We first thresholded the pre-exercise Vista scan at an intensity level commensurate with that of CSF ( $8 \mathrm{e}+5$ in this case). This reference scan was then precisely non-linearly registered to the subsequent scans with sub-voxel precision. The resulting images were thresholded at the same level and the sum of the voxel intensity values above the threshold was computed. This integral measure can then be used to compute differences across subject or before and after exercise. This approach was intended to alleviate the influence of changes in contrast, brightness and homogeneity across scans and the artefacts of partial voluming. A proxy for brain size was also obtained by linearly registering the reference scan to the ICBM standard atlas, with the product of the scaling factors along each axis used as a covariate in the statistical analysis. 
Paragraph 12 Blood handling and analysis. Blood samples (5 ml) were drawn by venous puncture from a superficial antecubital vein prior to the start of exercise, at the end of the dehydration protocol, and after 1 and 2 h of recovery. Subjects were seated for at least 15 min prior to each collection. Blood samples were drawn directly into dry syringes: a $1 \mathrm{ml}$ aliquot was dispensed into tubes containing $\mathrm{K}_{2}$ EDTA, with the remaining $4 \mathrm{ml}$ placed into a plain tube. Hemoglobin (in duplicate by the cyanmethaemoglobin method) and hematocrit (in triplicate by microcentrifugation) values were used to estimate percentage changes in blood, plasma and red cell volumes relative to the resting sample (6). The portion of the sample added to a plain tube was left to clot at room temperature for $60 \mathrm{~min}$ before being centrifuged to yield serum. This was kept at $4{ }^{\circ} \mathrm{C}$ for the analysis in duplicate of serum sodium, potassium (Corning 410C, New York, USA) and chloride (Jenway PCLM 3, Essex, UK) concentrations and serum osmolality (Gonotec Osmomat 030, YSI, Farnborough, UK).

Paragraph 13 Statistical Analysis Data are presented as means \pm standard deviation (SD) unless otherwise stated. To identify differences in normally distributed results, one-way repeated measures analysis of variance (ANOVA) was employed. Pair-wise differences were evaluated using paired t-tests with Holm-Bonferroni adjustment for multiple comparisons. Statistical significance was accepted at $\mathrm{P}<0.05$. Cohen's d effect sizes (ES) for the changes in brain, CSF and ventricular volumes were also determined [(Vol $\left.\left.\mathrm{Vre}-\mathrm{Vol}_{\text {post }}\right) / \mathrm{SD}\right]$. Regional changes in brain volume were determined by permutation-based, voxelwise non-parametric testing using Randomise (FMRIB Software Library, Oxford University, UK). Statistical inference was based 
on voxel-level thresholding at corrected P-values of 0.05 , adjusted for multiple comparisons.

\section{Results}

Paragraph 14 Dehydration data Pre-exercise urine osmolality was $495 \pm 116$ mosmol/kg and $449 \pm 145$ mosmol/kg during the familiarisation and experimental trials respectively $(\mathrm{P}=0.438)$. Total body mass loss was $2.31 \pm 0.29 \mathrm{~kg}$ during the familiarisation trial and $2.30 \pm 0.27 \mathrm{~kg}$ in the experimental trial, representing a $2.9 \pm$ $0.1 \%$ reduction in body mass. Subjects took $67.4 \pm 10.2 \mathrm{~min}$ of exercise to reach this level of hypohydration, with a total of $107 \pm 15$ min spent inside the climatic chamber.

Paragraph 15 Volumetric analysis While a full control trial was not included in the present investigation, normal variation in measured brain and CSF volumes were monitored over the same timeframe as the experimental trials in two subjects who did not participate in the main study. There was no change in brain (difference from scan 1: Scan2 $0.0 \pm 0.0 \%$, Scan3 $0.0 \pm 0.0 \%$ ) or CSF (difference from Scan 1: Scan 21.0 $\pm 0.3 \%$, Scan $30.2 \pm 0.0 \%$ ) volumes over the three hour rest period. Because of the limited sample size, it is difficult to draw definitive conclusions regarding the normal variation and/or measurement error at rest, but these values fall within the reported test/retest error of SIENA (0.2\% brain volume change; 20$)$. 
Paragraph 16 The change in brain and CSF volumes resulting from exercise/heatinduced hypohydration determined using SIENA are presented in Figure 1. Brain volume changes were unaffected by hypohydration (0.2 $\pm 0.4 \%$; ES 0.2; P = 0.310). A modest reduction in ventricular volume was observed following exercise $(4.0 \pm 1.8$ \%; ES 4.6; $\mathrm{P}<0.001$ ), with evidence of a gradual restoration over the $2 \mathrm{~h}$ recovery period. This response is highlighted in Figure 2, with significant morphometric edge flow of the brain/CSF boundary surrounding the ventricles indicating some degree of shrinkage between the pre-exercise and post-exercise scans and the pre-exercise and 2 $\mathrm{h}$ recovery scans $(\mathrm{P}<0.05)$. Employing analysis method 2 (vista scan), percent changes in brain and CSF volumes were also estimated (Figure 3). Again, brain volume was not influenced by hypohydration $(0.0 \pm 0.4 \%$; $\mathrm{P}=0.805)$, but in a similar manner to the ventricular volume data presented above, a small reduction in CSF volume was apparent following exercise $(3.1 \pm 1.9 \%$; $\mathrm{P}=0.003)$. This response was maintained throughout the $2 \mathrm{~h}$ rest period. There was a moderate relationship between the change in brain volume calculated using analysis methods 1 and $2\left(\mathrm{r}^{2}=\right.$ $0.611, \mathrm{P}=0.022$ ), with SIENA estimating a slightly greater change in brain volume following exercise in 7 of the 8 subjects.

Paragraph 17 Blood data Blood and plasma volumes were $9.0 \pm 2.5 \%$ and $13.7 \pm$ $3.9 \%$ lower respectively immediately after exercise, with some recovery of both volumes apparent during the recovery period $(\mathrm{P}<0.001$; Figure 4$)$. Serum sodium concentration increased from $144 \pm 1 \mathrm{mmol} / \mathrm{L}$ to $148 \pm 1 \mathrm{mmol} / \mathrm{L}$ during exercise $(\mathrm{P}=$ 0.003), but there was no change in serum potassium $(\mathrm{P}=0.062)$ or chloride $(\mathrm{P}=$ 0.210) concentrations (Table 1). Compared with pre-exercise levels, serum osmolality 
was increased at the end of exercise $(+10 \pm 2$ mosmol/kg; $\mathrm{P}<0.001)$ and remained elevated throughout the $2 \mathrm{~h}$ post-exercise period (Table 1).

Paragraph 18 Supplemental data Core temperature data obtained using a radiotelemetry pill are presented in Figure 5. Pre-exercise core temperature was $37.1 \pm 0.3$ ${ }^{\circ} \mathrm{C}$, with exercise resulting in a $2.2 \pm 0.4{ }^{\circ} \mathrm{C}$ increase. Core temperature at the end of exercise was $39.3 \pm 0.5{ }^{\circ} \mathrm{C}$, but returned to pre-exercise levels after $1 \mathrm{~h}$ of recovery. Subjective feelings related to thirst and hunger, tiredness and the ability to concentrate, and headache are presented in Table 2. There was a marked increase in perceived thirst reported immediately following exercise, with this maintained throughout the two-hour post-exercise period $(\mathrm{P}<0.001)$. There was no change in perceived feelings of tiredness $(\mathrm{P}=0.201)$ or the ability to concentrate $(\mathrm{P}=0.378)$, but the subjects reported a drop in alertness $(\mathrm{P}=0.037)$ and perceived energy $(\mathrm{P}=0.021)$ following exercise. Subjects reported no symptoms of headache before exercise, but feelings of head soreness were apparent following exercise and during recovery $(\mathrm{P}=$ $0.011)$.

\section{Discussion}

Paragraph 19 The results of the present study demonstrate that brain volume remains unchanged in response to moderate levels of acute hypohydration induced by exercise in a warm environment, but there do appear to be small reductions in ventricular and CSF volumes. Osmotically-driven movement of fluid from the CNS to the circulation appears to be minimal following exercise/heat-induced hypohydration, perhaps 
suggesting that mechanisms are in place to defend brain and CSF volumes in spite of a substantial increase in extracellular osmolality. The protocol employed in the present investigation resulted in the loss of $2.9 \pm 0.1 \%$ of the subjects' initial body mass: this level of hypohydration would be sufficient to reduce exercise capacity, particularly when exercise is performed in a warm environment (11).

Paragraph 20 The white and grey matter of the brain consist of approximately $70 \%$ and $82 \%$ water respectively (22) and brain volume is therefore susceptible to changes induced by fluctuations in water content. The water content of the brain is dictated largely by the solute content of the brain tissue relative to that of the extra-cerebral space, and a change in plasma osmolality can result in a net flux of water into the circulation. Several animal studies have reported marked changes in brain volume in response to peripheral infusions of hypertonic $\mathrm{NaCl}$ and mannitol that resulted in a state of hypernatremia $(1,3,4,9)$. Similarly, in the presence of hyponatremia, water can move from the circulation into the brain (along an osmotic gradient) resulting in cerebral edema (8). In the rat, acute hypernatremia results in a $7 \%$ reduction in total brain volume within 30-90 min, with the fluid being drawn primarily from the extracellular water compartments (4). These authors also reported that the degree of water loss from the brain in response to the osmotic challenge was not as great as predicted on the basis of ideal osmotic behaviour, and they attributed this to a net influx of electrolytes from the cerebrospinal fluid and plasma $(1,3)$. This volume regulation acts as a defense mechanism that limits the degree of brain shrinkage $(1,8)$. It should be noted, however, that the hypernatremia induced in the nephrectomised rats studied by Cserr et al. (4) was particularly severe, with plasma sodium concentrations increasing by $30 \mathrm{mmol} / \mathrm{L}$. This is far beyond the extent of change that 
would occur in healthy humans during prolonged exercise, when sodium concentration seldom increases by more than about 5-6 mmol/L. In a group of 90 marathon runners, for example, the mean increase in plasma sodium concentration over the course of the race was $6 \mathrm{mmol} / \mathrm{l}(26)$.

Paragraph 21 At present there are limited data on the effects of changes in fluid balance on the CNS in humans but three recent studies employing scanning techniques have examined changes in brain and ventricular volumes with hypohydration $(5,7,10)$. A 16 hour period of fluid restriction, resulting in a body mass loss of $1.6 \pm 1.0 \%$, produced a significant reduction in total brain volume. Brain volume was subsequently restored by rehydration with plain water (7). Employing various degrees of exercise and heat exposure, Dickson and colleagues (5) and Kempton et al. (10) reported no reduction in total brain volume, but they found marked changes in ventricular volume with levels of hypohydration between 1.7 and $2.9 \%$. The striking outcome of both these exercise studies was the marked variation in ventricular volume change observed following exercise (changes from $-15 \%$ to +42 $\%)$, with the magnitude and direction of change related to the degree of hypohydration induced. It should be noted that the extent of fluid loss induced in these studies was highly variable, with the subjects who attained body mass losses of more than $2.5 \%$ displaying ventricular expansion, a response which is difficult to explain. Subjects in the present study all lost between $2.8 \%$ and $3.1 \%$ of body mass, but none of these subjects showed an increase in ventricular volume. The present data suggest that brain volume is tightly maintained in the presence of marked reductions in total body water, and these distinct and variable changes in ventricular or CSF volumes were not apparent. 
Paragraph 22 The present study found a $3.1 \pm 1.9 \%$ reduction in CSF volume in the presence of moderate levels of hypohydration. Ventricular volume was also reduced immediately following exercise-induced hypohydration, with a gradual restoration of this loss apparent over the 2 hour recovery period. While these differences were statistically significant, it is unlikely that a change of this magnitude is physiologically relevant, with the change in CSF volume representing a fluid loss of only 4-5 mL from this compartment, compared with the larger changes in blood $(9 \pm$ 3\%) and plasma (14 $\pm 4 \%$ ) volumes. This represents a loss of about 410 to $600 \mathrm{ml}$ of water from the vascular space, based on the assumption that blood volume was 5582 $\pm 786 \mathrm{ml}$ in these subjects (blood volume estimates based on $70 \mathrm{ml} / \mathrm{kg}$ body mass; 24). Regional changes in brain and ventricular volumes were highlighted using the voxelwise extension of SIENA. Both methods employed to estimate differences in brain volume reported no global volume change, but there was a suggestion of some regional redistribution of fluid within the brain following exercise and heat-induced hypohydration (Figure 2). The physiological importance of these changes are unknown at present, but this may be implicated in the decrements in several aspects of cognitive function reported following strenuous exercise in warm conditions.

Paragraph 23 The technical challenges associated with the measurement of changes in brain, ventricular and CSF volumes using MRI techniques should be recognised. The cerebrospinal fluid is produced by the ependymal cells of the choroid plexus found throughout the ventricular system and provides basic mechanical and immunological protection to the brain inside the skull, as well as a transport medium for nutrients and neurotransmitters. CSF flows from the lateral ventricles into the third ventricle and then the fourth ventricle via the cerebral aqueduct in the brainstem. The 
aqueduct between ventricles is small and presents a common source of error when determining ventricular and CSF volumes. The application of two separate volumetric analysis techniques, with both producing similar outcomes, should provide additional confidence in the data presented. It is difficult to explain the marked discrepancy in changes in ventricular volume reported between the present study and those published previously $(5,10)$, but it is possible that the variable levels of hypohydration attained in the earlier studies might be a factor, along with differences in the timeframe between the end of exercise and the completion of the MRI scan.

Paragraph 24 In the present study, serum osmolality increased by $10 \pm 2 \mathrm{mosmol} / \mathrm{kg}$ ( $\mathrm{P}<0.001)$ during exercise, with mean values of $294 \pm 2 \mathrm{mosmol} / \mathrm{kg}$ observed at the end of exercise. This is similar to the response observed in a previous study investigating changes in serum S100 $\beta$ concentrations during intermittent exercise in the heat, where serum osmolality increased by $12 \pm 3 \mathrm{mosmol} / \mathrm{kg}$ (25). While this change is small relative to that produced by mannitol or glycerol infusion (which can result in serum osmolality values in excess of $310 \mathrm{mosmol} / \mathrm{kg}$ ), the resulting osmotic gradient between the periphery and CNS should have been sufficient to produce a net movement of fluid from the brain to the circulation. As highlighted previously, the degree of brain water loss observed in animals exposed to acute hyperosmotic states was not as great as predicted on the basis of ideal osmotic behaviour (3). The accumulation of solutes in the brain, including electrolytes drawn from the CSF and plasma, occurs during acute hyperosmotic states to limit fluid losses. This volume regulation occurs rapidly and acts to restrict brain shrinkage $(1,8)$. It appears that this mechanism is in operation when individuals are exposed to moderate hypohydration resulting from exercise in warm/humid conditions. 
Paragraph 25 This movement of fluids might protect against potential changes in brain volume resulting from the dehydration practices used by competitors in some sports where a significant time interval exists between the weigh-in and the start of competition (e.g. professional boxing). There are cases, however, where competition begins shortly following a weigh in (horse racing, amateur boxing/martial arts): this, coupled with any hypohydration accrued during the activity itself, could place these individuals at an increased risk of traumatic brain injury. The convulsive theory of concussion suggests that an abrupt increase or sudden arrest of head movement, results in an angular acceleration of the head that causes turbulent rotation of the mass of brain within the cranium (18). Sudden mechanical loading of the head might increase the chances of a tissue-deforming collision between the brain cortex and the walls of the skull. Any reduction in the volume of the brain, or in the volume of CSF surrounding the brain, might increase trauma experienced during falls and collisions because of a greater degree of brain movement within the skull.

Paragraph 26 It is important to recognise the limitations of this study and of other MRI-based studies that have examined changes in brain volume. It is clear that mechanisms operate to limit fluid losses from the brain, and the magnitude of brain water losses has been demonstrated to be only about $35 \%$ of that expected $30 \mathrm{~min}$ following infusion of hyperosmotic solutions (3). Subjects were positioned in the MRI magnet within 5 min after the end of exercise, and the scanning procedure took around 10 min to complete. Despite efforts to ensure a rapid turnaround, water loss and electrolyte uptake are likely to occur simultaneously, potentially causing part of any response to be missed. Animal studies investigating the effect of dehydration by $10 \%$ of initial body mass also report no change in brain volume, but these 
measurements were taken once core temperature had returned to basal levels (14). Again, it is probable that there was a redistribution of fluids during this time to restore brain volume. It is also important to acknowledge the likely influence of posture change on the results presented. Moving from a standing to supine position results in a marked redistribution of fluid within the body, with blood and plasma volumes increasing by 8 and $17 \%$ respectively following $60 \mathrm{~min}$ in the supine position (16). It is possible that moving from a seated position whilst at rest and during exercise to a supine position in the scanner could have distorted the results, but this same artefact should be present in all scans of this nature unless the posture of the subjects is first stabilised and then maintained throughout the entire study period.

Paragraph 27 In conclusion, these data demonstrate that brain volume remains unchanged in response to moderate levels of hypohydration, but there does appear to be a small transient reduction in CSF volume. Osmotically-driven movement of fluid from the CNS to the circulation appears to be minimal following exercise/heatinduced hypohydration, perhaps suggesting that mechanisms are in place to defend brain volume.

\section{Acknowledgements}

The authors would like to acknowledge the Gatorade Sport Science Institute (GSSI), Barrington, Illinois, USA for providing financial support for this study. The results of the present study do not constitute endorsement by ACSM. 


\section{References}

1 Ayus JC, Armstrong DL, Arieff AI. Effects of hypernatraemia in the central nervous system and its therapy in rats and rabbits. $J$ Physiol. 1996;492:243-55.

2 Bledsoe GH, Li G, Levy F. Injury risk in professional boxing. South Med J. 2005;98:994-998.

3 Cserr HF, DePasquale M, Patlak CS. Regulation of brain water and electrolytes during acute hyperosmolality in rats. Am J Physiol. 1987;253:F522-529.

$4 \quad$ Cserr HF, DePasquale M, Nicholson C, Patlak CS, Pettigrew KD. Extracellular volume decreases while cell volume is maintained by ion uptake in rat brain during acute hypernatremia. J Physiol. 1991;442:277-295.

5 Dickson JM, Weavers HM, Mitchell N, Winter EM, Wilkinson ID, Van Beek EJ, Wild JM, Griffiths PD. The effects of dehydration on brain volume preliminary results. Int J Sports Med. 2005;26:481-485.

6 Dill DB, Costill DL. Calculation of percentage changes in volumes of blood, plasma, and red cells in dehydration. J Appl Physiol. 1974;37:247-248. 
$7 \quad$ Duning T, Kloska S, Steinstrater O, Kugel H, Heindel W, Knecht S. Dehydration confounds the assessment of brain atrophy. Neurology 2005;64:548-550.

8 Gullans SR, Verbalis JG. Control of brain volume during hyperosmolar and hypoosmolar conditions. Ann Rev Med. 1993;44:289-301.

9 Holliday MA, Kalayci MN, Harrah J. Factors that limit brain volume changes in responses to acute and sustained hyper- and hyponatremia. J Clin Invest. 1968;47:1916-1928.

10 Kempton MJ, Ettinger U, Schmechtig A, Winter EM, Smith L, McMorris T, Wilkinson ID, Williams SC, Smith MS. Effects of acute dehydration on brain morphology in healthy humans. Hum Brain Mapp. 2009;30:291-298.

11 Maughan RJ, Shirreffs SM. Development of individual hydration strategies for athletes. Int J Sport Nutr Exerc Metab. 2008;18:457-72.

12 Moore JM, Timperio AF, Crawford DA, Burns CM, Cameron-Smith D. Weight management and weight loss strategies of professional jockeys. Int $J$ Sport Nutr Exerc Metab. 2002;12:1-13.

13 Nose H, Mack GW, Shi X, Nadel ER. Shift in body fluid compartments after dehydration in humans. J Appl Physiol. 1988;65:318-324. 
14 Nose H, Morimoto T, Ogura K. Distribution of water losses among fluid compartments of tissues under thermal dehydration in the rat. Jpn J Physiol. 1983;33:1019-1029.

15 Rueckert D, Sonoda LI, Hayes C, Hill DLG, Leach MO, Hawkes DJ. Nonrigid registration using free-form deformations: Application to breast MR images. IEEE Transactions on Medical Imaging. 1999;18:712-721.

16 Shirreffs SM, Maughan RJ. The effect of posture change on blood volume, serum potassium and whole body electrical impedance. Eur J Appl Physiol. 1994;69:461-3.

17 Shirreffs SM, Maughan RJ. Urine osmolality and conductivity as indices of hydration status in athletes in the heat. Med Sci Sports Exerc. 1998;30:1598602.

18 Shaw NA. The neurophysiology of concussion. Prog Neurobiol. 2002;67:281344.

19 Smith SM. Fast robust automated brain extraction. Hum Brain Mapp. 2002;17:143-155.

20 Smith SM, Zhang Y, Jenkinson M, Chen J, Matthews PM, Federico A, De Stefano N. Accurate, robust and automated longitudinal and cross-sectional brain change analysis. NeuroImage 2002;17:479-489. 
21 Smith SM, Jenkinson M, Woolrich MW, Beckmann CF, Behrens TEJ, Johansen-Berg H, Bannister PR, De Luca M, Drobnjak I, Flitney DE, Niazy R, Saunders J, Vickers J, Zhang Y, De Stefano N, Brady JM, Matthews PM. Advances in functional and structural MR image analysis and implementation as FSL. NeuroImage 2004;23:208-219.

22 Toffs PS. PD: Proton density of tissue water. In: Toffs PS, editor. Quantitative MRI of the Brain: Measuring Changes Caused by Disease. Malden MA, USA: Wiley; 2000. p. 85-110.

23 Turner M, McCrory P, Halley W. Injuries in professional horse racing in Great Britain and the Republic of Ireland during 1992-2000. Br J Sports Med. 2002;36:403-409.

24 Wasserman LR, Yoh T, Rashkoff IA. Blood volume determination: comparison of T-1824 and P32 labelled red cell methods. J Lab Clin Med. $1951 ; 37: 342-52$

25 Watson P, Black KE, Clark SC, Maughan RJ. Exercise in the heat: effect of fluid ingestion on blood-brain barrier permeability. Med Sci Sports Exerc. 2006;38:2118-2124.

26 Whiting PH, Maughan RJ, Miller JDB. Dehydration and serum biochemical changes in marathon runners. Eur J Appl Physiol. 1984;52:183-187. 
27 Zhang Y, Brady M, Smith S. Segmentation of brain MR images through a hidden Markov random field model and the expectation maximization algorithm. IEEE Trans Med Imag. 2001;20:45-57. 


\section{Figure Legends}

Figure 1: The percentage change in brain (top) and ventricular (bottom) volumes following exercise/heat-induced hypohydration calculated using analysis method 1 (SIENA). ${ }^{* *}(\mathrm{P}<0.01)$ denotes a significant difference from the Pre-Ex value.

Figure 2: Voxelwise statistical analysis highlighting significant perpendicular displacement of the brain/CSF boundary between the pre-exercise and post-exercise scans (A) and the pre-exercise and $2 \mathrm{~h}$ recovery scans (B). Red colored regions indicate volume loss at a $\mathrm{P}=0.05$ level, with regions where the color graduates to white indicating brain volume shrinkage at a $\mathrm{P}<0.01$ level.

Figure 3: The percentage change in brain (top) and CSF (bottom) volumes following exercise/heat-induced hypohydration calculated using analysis method 2. ** $(\mathrm{P}<$ 0.01) denotes a significant difference from the Pre-Ex value.

Figure 4: Change in plasma volume following exercise/heat-induced hypohydration. $*(\mathrm{P}<0.05)$ and $* *(\mathrm{P}<0.01)$ denote a significant difference from the Pre-Ex value.

Figure 5: Core temperature during exercise and recovery. * $(\mathrm{P}<0.05)$ and $* *(\mathrm{P}<$ 0.01) denote a significant difference from the Pre-Ex value. 

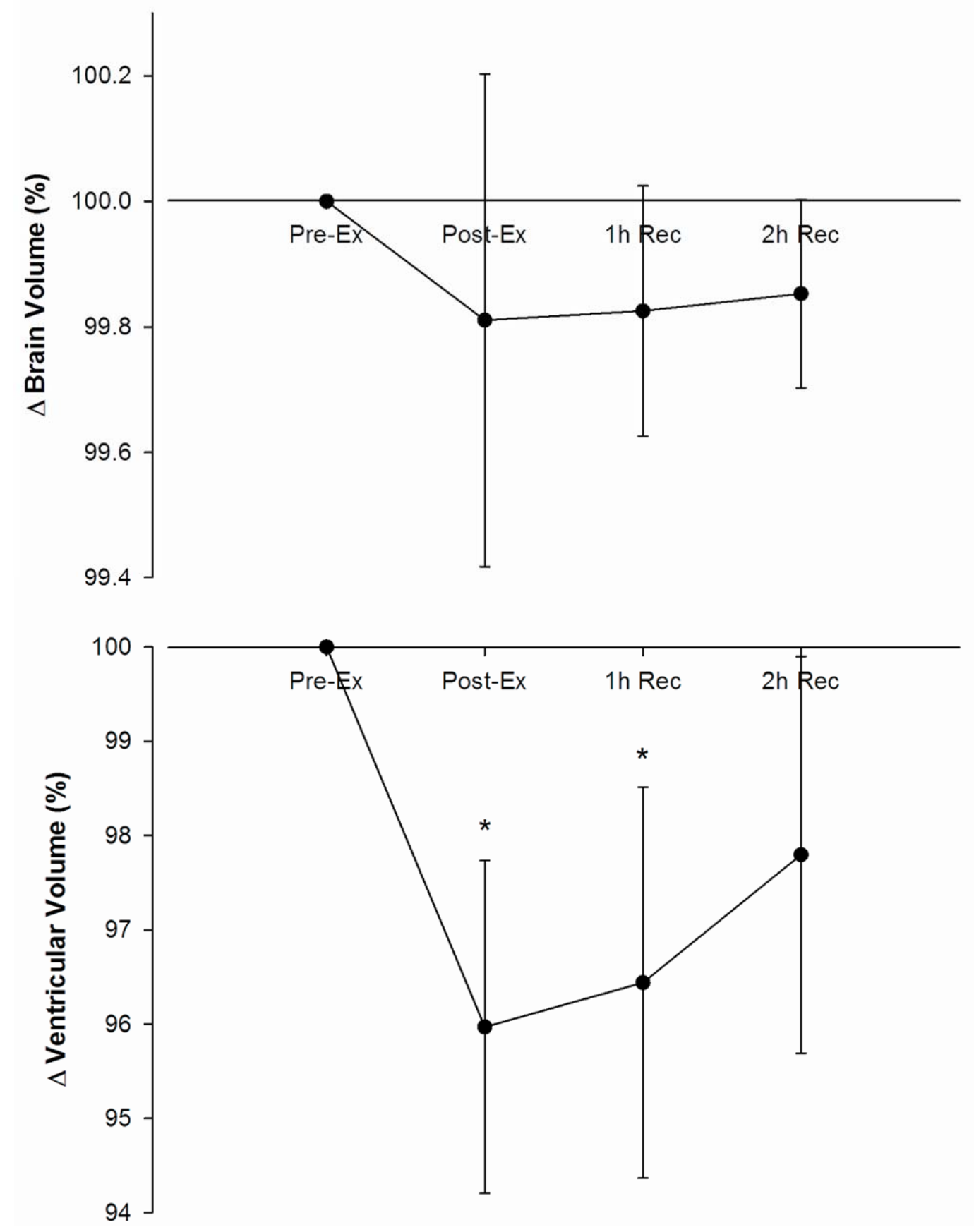


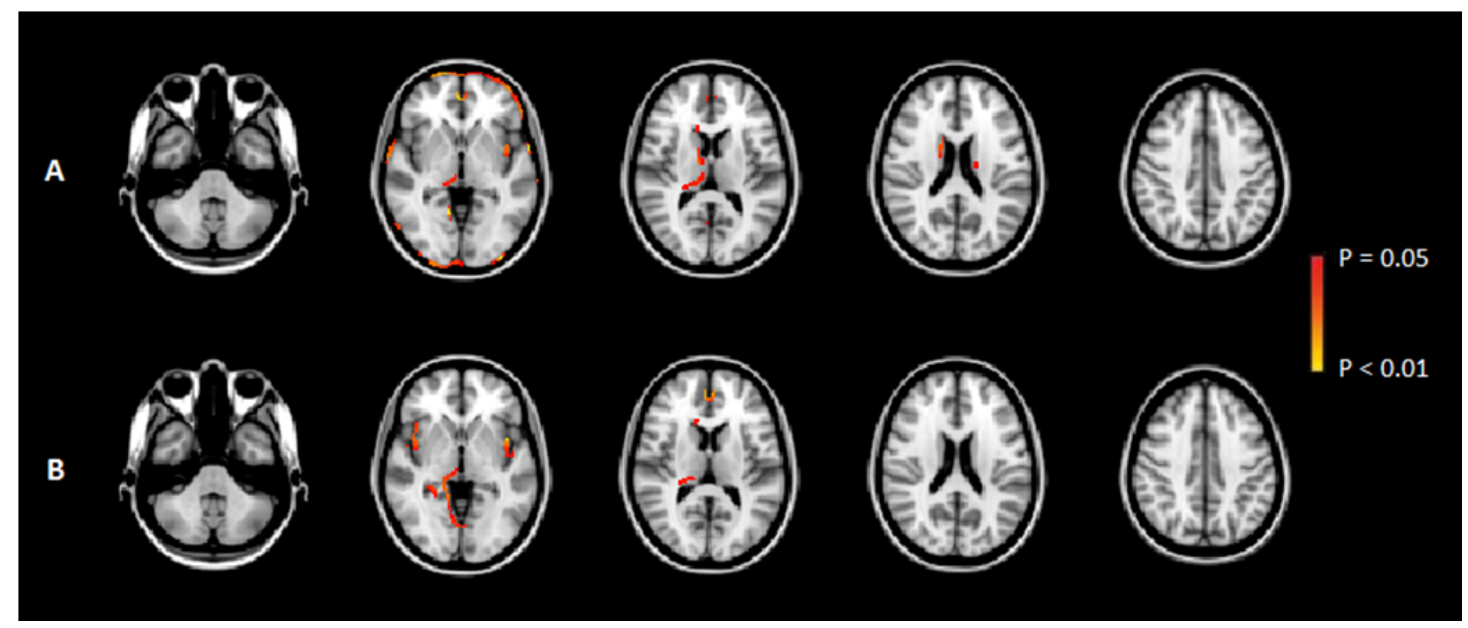



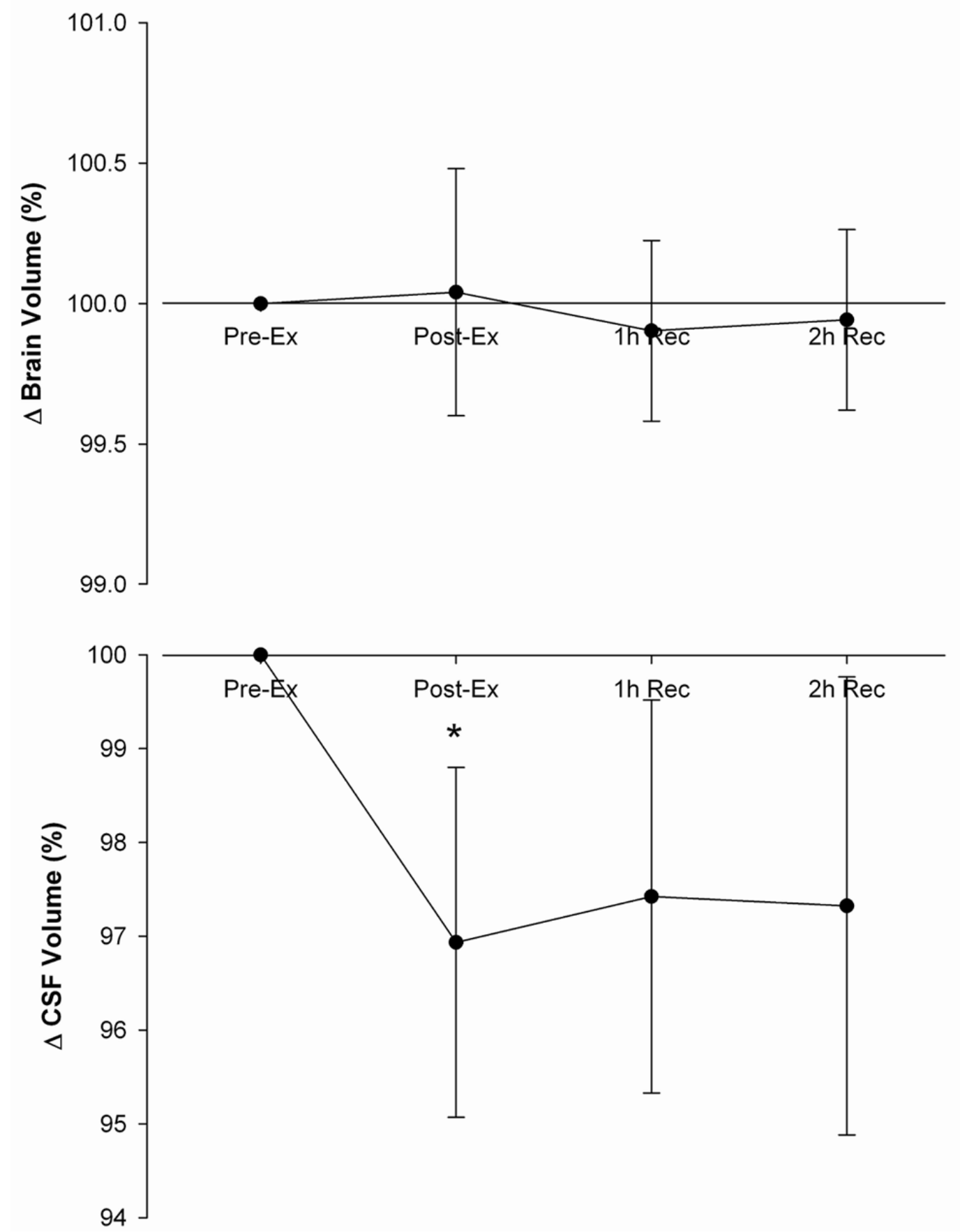


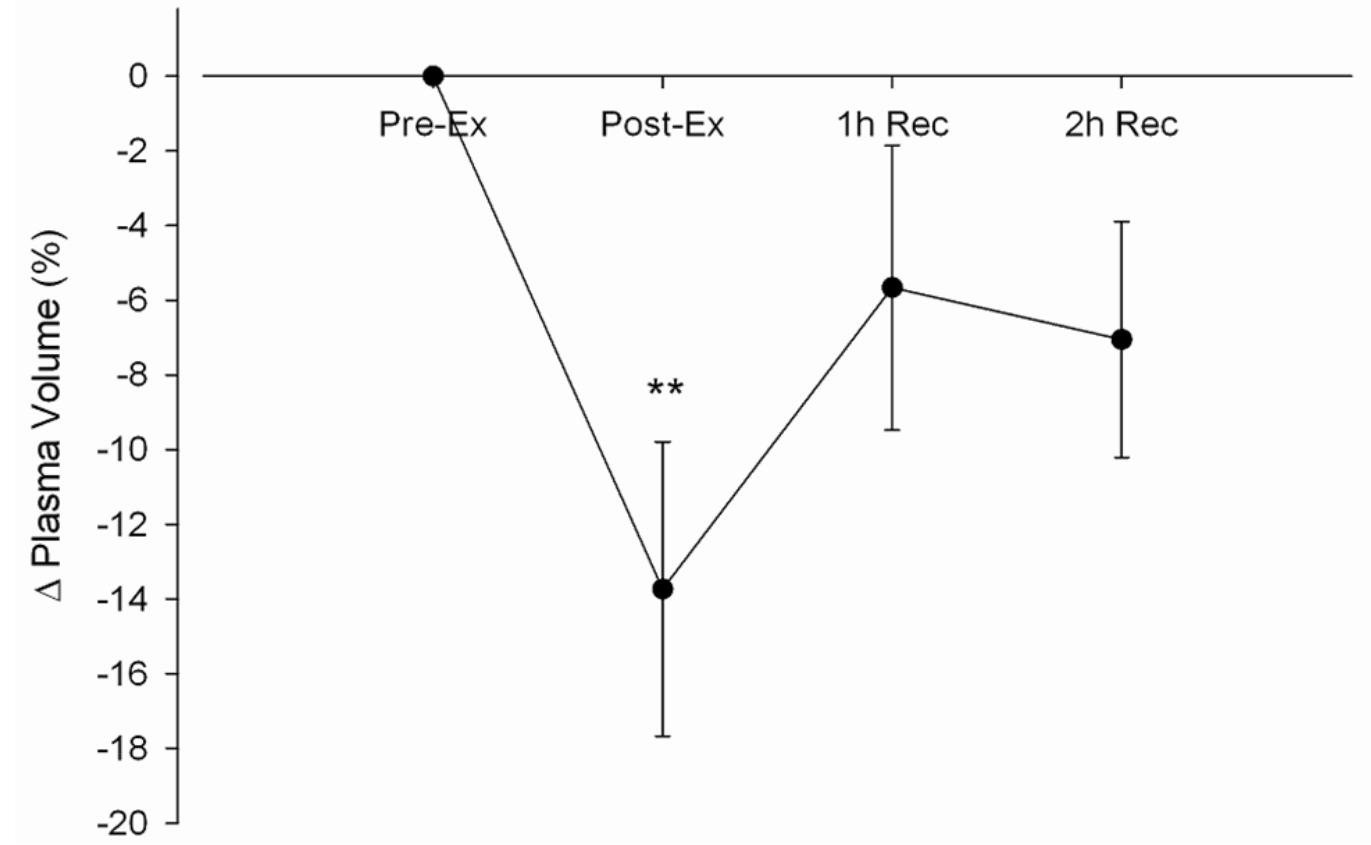




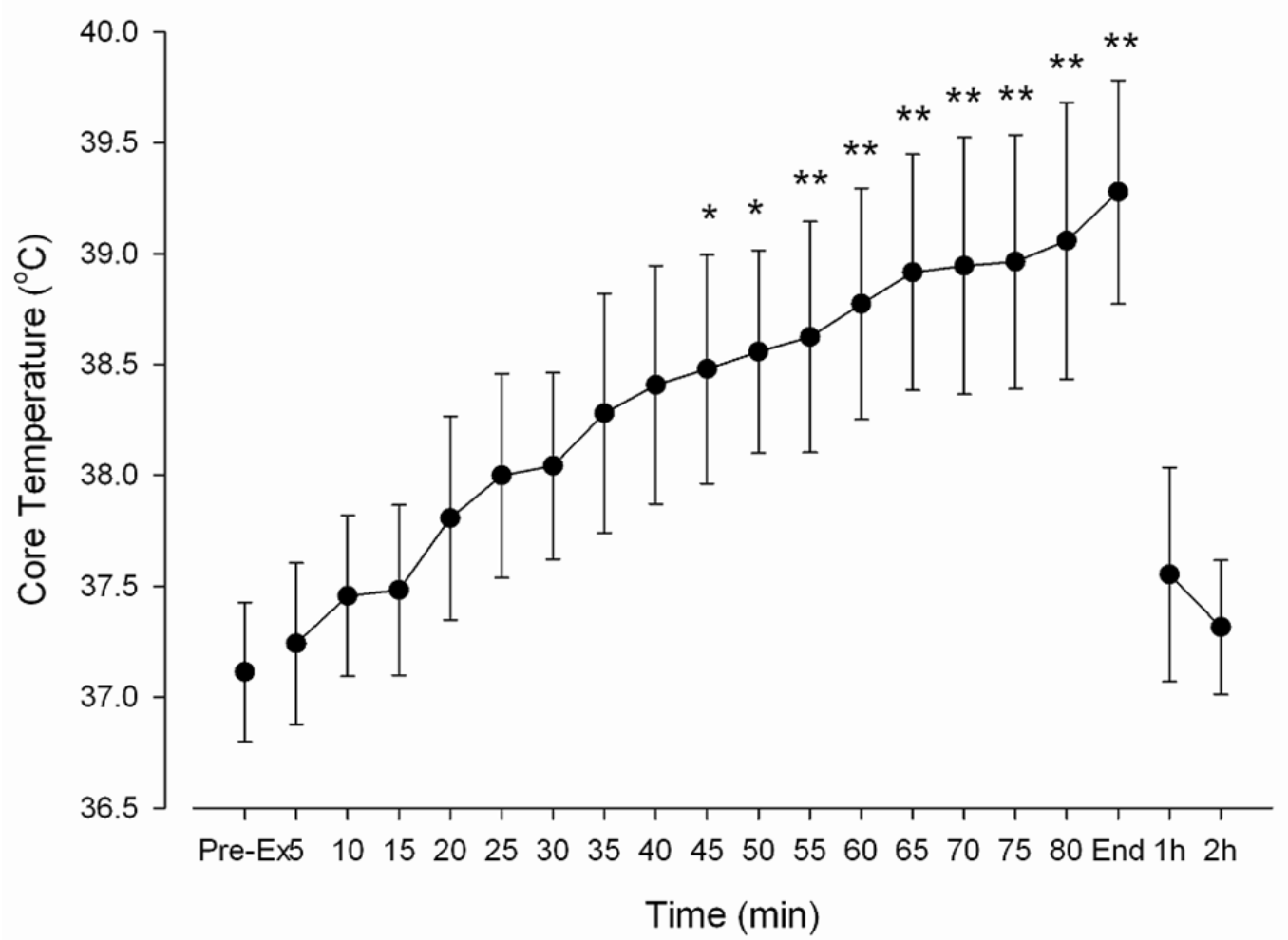


Table 1: Serum electrolyte concentrations and osmolality. ** $(\mathrm{P}<0.01)$ denotes a significant difference from the Pre-Ex value.

\begin{tabular}{lcccc}
\hline & Pre-Ex & Post-Ex & 1h rec & 2h rec \\
\hline Sodium (mmol/L) & $144 \pm 1$ & $148 \pm 1^{* *}$ & $147 \pm 1$ & $146 \pm 1$ \\
Potassium (mmol/L) & $4.6 \pm 0.2$ & $5.2 \pm 0.5$ & $4.7 \pm 0.4$ & $4.6 \pm 0.3$ \\
Chloride (mmol/L) & $98 \pm 2$ & $100 \pm 3$ & $99 \pm 3$ & $98 \pm 3$ \\
Osmolality (mosmol/kg) & $284 \pm 2$ & $294 \pm 2^{* *}$ & $290 \pm 3^{* *}$ & $290 \pm 4$ \\
\hline
\end{tabular}

Table 2: Subjective feelings related to thirst, tiredness, the ability to concentrate, and headache (scored on a $100 \mathrm{~mm}$ scale). $*(\mathrm{P}<0.05)$ and $* *(\mathrm{P}<0.01)$ denote a significant difference from the Pre-Ex value.

\begin{tabular}{lllll}
\hline & Pre-Ex & Post-Ex & 1h rec & 2h rec \\
\hline Thirst & $39 \pm 19$ & $78 \pm 22^{* *}$ & $85 \pm 14^{* *}$ & $88 \pm 14^{* *}$ \\
Tiredness & $57 \pm 9$ & $69 \pm 14$ & $54 \pm 32$ & $68 \pm 22$ \\
Alertness & $46 \pm 23$ & $35 \pm 12^{*}$ & $39 \pm 26$ & $33 \pm 26$ \\
Concentration & $39 \pm 14$ & $31 \pm 19$ & $32 \pm 20$ & $34 \pm 27$ \\
Energy & $55 \pm 13$ & $29 \pm 7^{* *}$ & $36 \pm 18^{*}$ & $35 \pm 21^{*}$ \\
Headache & $4 \pm 3$ & $56 \pm 29^{* *}$ & $53 \pm 30^{* *}$ & $57 \pm 31^{* *}$ \\
\hline
\end{tabular}

